\title{
Dynamic Behavior of Low Impedance Materials
}

\author{
J. L. Jordan ${ }^{1}$ - C. R. Siviour ${ }^{2}$ - B. Song ${ }^{3}$
}

Published online: 1 February 2016

(C) Society for Experimental Mechanics, Inc 2016 (outside the US) 2016

The dynamic behavior of low impedance materials is an extremely active area of research; however, special consideration must be taken when performing experiments on these materials, due to particular aspects of their response at high strain rates. Materials that may be considered low impedance include polymers, foams, biological materials, and composites. The experimental techniques and diagnostics used at high strain rates may vary from those required for more established dynamic testing, for example of metals due to the low response signals generated by low impedance materials, and the low sound speed.

In order to highlight the unique properties and experimental techniques for these materials, since 2005, sessions with a focus on the dynamic behavior of low impedance materials have been organized by the Dynamic Behavior of Materials Technical Division at the annual Society for Experimental Mechanics conferences. Based on the success of these technical sessions and due to the interest in this topic within the dynamic behavior of materials community, this special issue of the Journal of the Dynamic Behavior of Materials is dedicated to the experimental techniques and properties of this class of materials.

The special issue covers all aspects of dynamic behavior of low impedance materials from the broad international community. Two review articles covering the state-of-the-art in low-impedance-material characterization (Chen) and the high-strain-rate properties of polymers (Siviour and Jordan) provide an overview of important aspects of the field. Advancements in experimental techniques are addressed by Govender \& Curry and Cloete \& Prot. Finally, studies on a wide variety of low impedance materials including biological materials by Luo, Jiang, Nakmali, Gan \& Lu and Weerasooriya, Sanborn, Gunnarsson \& Foster; polymers by Jordan, Montaigne, Gould, Neel, Sunny \& Molek, Wang, Bahlouli, Boumbimba, Addiego \& Rémond, Foster, Masser \& Lenhart, Bourne, and Trumiño and Cronin; foams by Sanborn, Song, and Smith; and composites by Ravindran, Tessema \& Kidane and Boddy, Gould, Jardine \& Williamson.

The guest editors gratefully acknowledge the technical contributions of the authors and the dedicated efforts of the reviewers for making this special issue possible. We are particularly thankful to Dr. Eric Brown, Editor-in-Chief of Journal of Dynamic Behavior of Materials, for his expertise and patient guidance through the entire process. We also want to thank Mr. Nuno Lopes, Managing Editor of Journal of Dynamic Behavior of Materials and the entire Society for Experimental Mechanics staff for making the issue possible.

\footnotetext{
J. L. Jordan

jennifer.jordan.6@us.af.mil

1 Air Force Office of Scientific Research, Arlington, VA 22203, USA

2 Department of Engineering Science, University of Oxford, Oxford OX13PJ, UK

3 Sandia National Laboratories, Albuquerque, NM 87185, USA
} 